\title{
Images of the month: Verruciform xanthoma: an easily confused disease
}

\author{
Authors: Qian An, ${ }^{\mathrm{A}}$ Li Zhang, ${ }^{\mathrm{B}}$ Xing-Hua Gao ${ }^{\mathrm{C}}$ and Song Zheng ${ }^{\mathrm{D}}$
}

Verruciform xanthomas occur primarily in the mouth, but sometimes in the anogenital region, which may cause clinical confusion with genital warts. We present a case where a 70-year-old man presented with an asymptomatic warty lesion on the scrotum and verruciform xanthoma was confirmed by histopathological results.

KEYWORDS: Verruciform, xanthoma

DOI: $10.7861 /$ clinmed.2019-0477

\section{Case presentation}

A 70-year-old man presented with an asymptomatic warty lesion on the scrotum, which initially developed as a small papule and enlarged into a polypoid nodule over 3 years. On examination, an exophytic yellowish verrucous nodule, measuring $3 \mathrm{~cm}$ in diameter, was found on the scrotum (Fig 1). The lesion was painless and moveable from the underlying structures. His past medical history was unremarkable and had no prior similar lesions on other sites.

\section{Investigations}

Laboratory investigation for biochemical panel including serum lipid profile was within normal range. An excisional biopsy specimen revealed numerous foamy histiocytes in the papillary dermis (Fig 2a) which were positive for CD68 immunohistochemical staining (Fig 2b). Detection of human papillomavirus (HPV) deoxyribonucleic acid by polymerase chain reaction was negative.

Based on these findings, the diagnosis of verruciform xanthoma was made. The patient received a complete surgical excision of the lesion and had no recurrence at a 6 -month follow-up.

Authors: A attending doctor, The First Hospital of China Medical University, Shenyang, China; ${ }^{B}$ associate professor, The First Hospital of China Medical University, Shenyang, China; ' professor, The First Hospital of China Medical University, Shenyang, China; ${ }^{D}$ associate professor of dermatology, The First Hospital of China Medical University, Shenyang, China

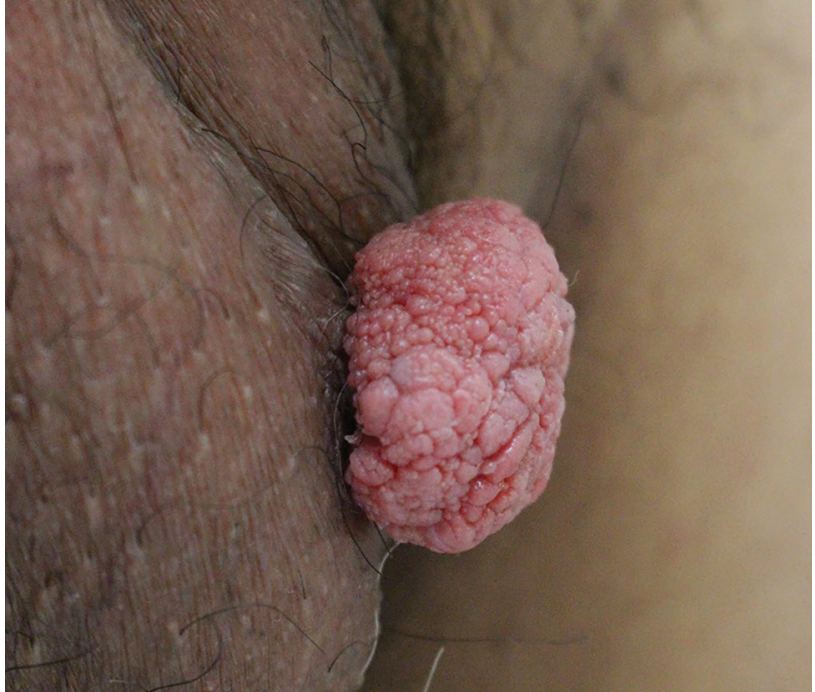

Fig 1. An exophytic yellowish verrucous nodule, measuring $3 \mathrm{~cm}$ in diameter, was found on the scrotum.

\section{Discussion}

The exact aetiology and pathogenesis of verruciform xanthoma remains unknown. Several hypotheses have been proposed, including HPV infections or local release of lipids by damaged keratinocytes through chronic inflammation, though no HPV were detected in our patient. ${ }^{1}$ In contrast to other forms of xanthomas, there is usually no associated hyperlipidemia in verruciform xanthoma cases, as with our case.

Verruciform xanthomas occur primarily in the mouth, but sometimes in the anogenital (including the scrotum) region, which may cause clinical confusion with genital warts. ${ }^{2}$

Verruciform xanthomas should be differentiated with verrucous carcinoma, squamous cell carcinoma, bowenoid papulosis, granular cell tumor, giant molluscum contagiosum, verruca vulgaris and vulvar intraepithelial neoplasia. Therefore, the diagnosis of verruciform xanthomas should be confirmed by the histopathological results. 

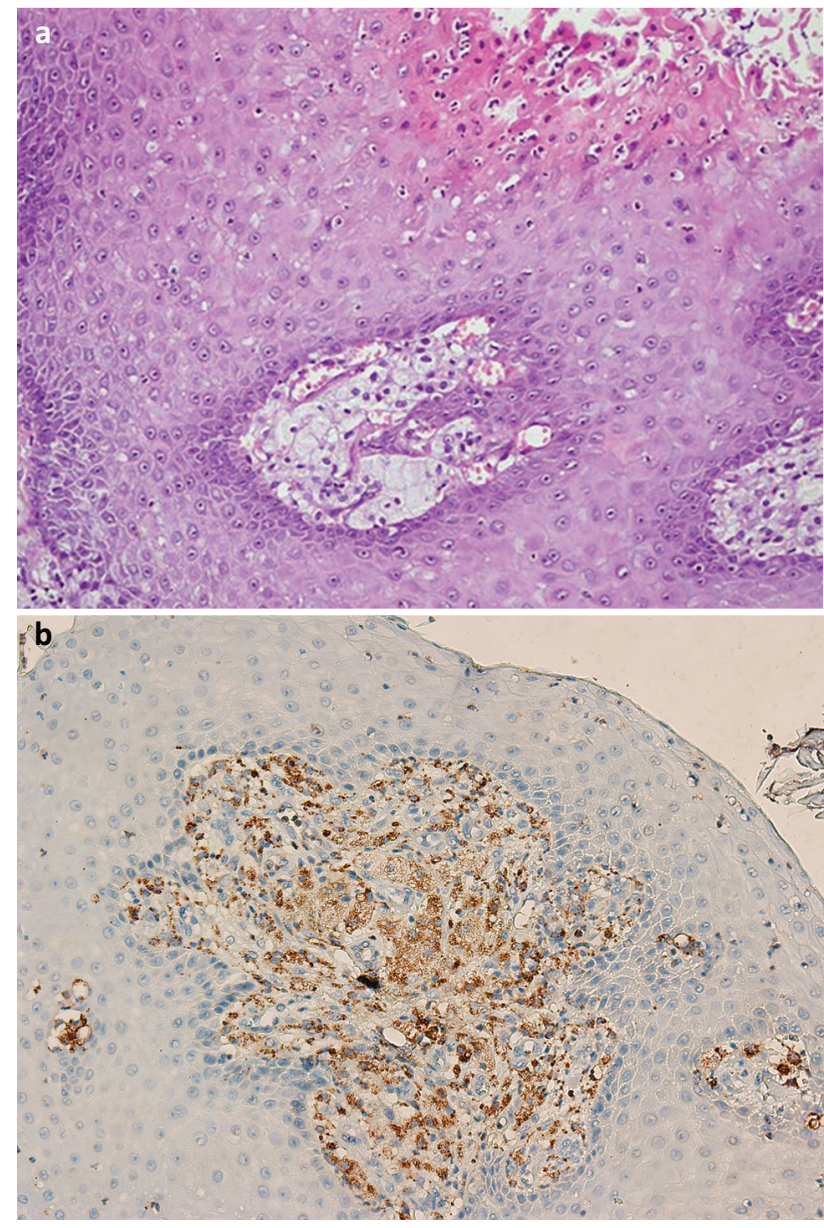

Fig 2. a) An excisional biopsy specimen revealed numerous foamy histiocytes in the papillary dermis. b) Foamy cells were positive for CD68 immunohistochemical staining.

\section{References}

1 Stiff KM, Cohen PR. Vegas (verruciform genital-associated) xanthoma: a comprehensive literature review. Dermatol Ther 2017;7: 65-79.

2 Joo J, Fung MA, Jagdeo J. Successful treatment of scrotal verruciform xanthoma with shave debulking and fractionated carbon dioxide laser therapy. Dermatol Surg 2014;40:214-7.

Address for correspondence: Assoc Prof Song Zheng, Department of Dermatology, The First Hospital of China Medical University, Shenyang 110001, China.

Email: nyaadzs@163.com 